\title{
SELF-CONSISTENT MODELLING OF PULSAR MAGNETOSPHERES
}

\author{
H. Herold ${ }^{1,2}$, T. Ertl ${ }^{2}$, B. Finkbeiner ${ }^{2}$, H. Ruder ${ }^{2}$ \\ ${ }^{1}$ Institut für Theoretische Physik der Universität Zürich \\ Schönberggasse 9 \\ $\mathrm{CH}-8001$ Zürich \\ Switzerland \\ ${ }^{2}$ Lehrstuhl für Theoretische Astrophysik der Universität Tübingen \\ Auf der Morgenstelle 12 \\ D-7400 Tübingen 1 \\ F.R.G.
}

\begin{abstract}
The magnetosphere of a rapidly rotating, strongly magnetized neutron star with aligned magnetic and rotational axes (parallel rotator) is modelled numerically. Including the radiation of the particles accelerated to relativistic energies as an efficient damping mechanism, we obtain a quasi-stationary selfconsistent solution to this classical problem. The numerical simulation, which was started from the well-known vacuum solution, yields a global magnetospheric structure that can be characterized by two regions of oppositely charged particles, which eventually produce a relativistic pulsar wind, separated by a vacuum gap of considerable extent.
\end{abstract}

\section{INTRODUCTION}

The problem - fundamental to the physics of pulsars - of determining the global structure of the magnetospheres of rapidly rotating strongly magnetized neutron stars has not yet been solved self-consistently (for a review cf. Michel 1982), but it must be solved before any conclusive interpretation can be given to the large amount of detailed experimental data on pulsar radiation (cf. e.g. the contribution by D. Backer in these proceedings). Apart from this reason, it is "... just intolerable that we should not know how a rotating magnetized neutron star comes to terms with its environment" (Mestel 1981).

We concentrate here on the self-consistent modelling of the magnetosphere of an aligned rotator, where the rotation axis is parallel to the magnetic axis of the neutron star. In this case, the homopolar induction, which should be responsible for populating the magnetosphere with charged particles pulled out from the neutron star surface via field emission (Goldreich and Julian 1969), can be studied in purity, whereas electromagnetic wave effects are neglected. The main questions 
to be answered are the following: What is the global structure of the electric and the magnetic field? How are the charge and current density distributed that produce these fields (together with the star)? Which particle populations exist in the magnetosphere?

\section{BASIC EQUATIONS}

\subsection{Maxwell and Vlasov Equations}

In the stationary axially symmetric case the electromagnetic fields $\mathbf{E}$ and $\mathrm{B}$ in the pulsar magnetosphere can be described (in cylindrical coordinates $\rho, \varphi, z$ ) by the electrostatic potential $\Phi(\rho, z)$, the magnetic flux function $\Psi(\rho, z)$ and the poloidal magnetic field $B_{\varphi}(\rho, z): \mathbf{E}=-\nabla \Phi$ and $\mathbf{B}=(1 / \rho) \nabla \Psi \times \mathbf{e}_{\varphi}+B_{\varphi} \mathbf{e}_{\varphi}$. The charge density $\rho_{e}$ and the current density $\mathbf{j}$ determine the electric potential via the Poisson equation and the magnetic field via Ampere's law, which here read in suitable units such that all quantities are dimensionless:

$$
\begin{aligned}
\Delta \Phi=-\rho_{e} ; & \left(\Delta-\frac{2}{\rho} \frac{\partial}{\partial \rho}\right) \Psi=-\rho j_{\varphi} \\
\frac{\partial}{\partial z}\left(\rho B_{\varphi}\right)=-\rho j_{\rho} ; & \frac{\partial}{\partial z}\left(\rho B_{\varphi}\right)=-\rho j_{z}
\end{aligned}
$$

The magnetosphere is formed by a collisionless plasma, in which the particles are expected to be extremely relativistic due to the huge electric and magnetic fields (the homopolar induction voltage between pole and equator of the neutron star is typically of the order $10^{17}-10^{18} \mathrm{~V}$ ). The charge and current densities are derived from the zeroth and first momentum of the particle distribution function

$$
\rho_{e}=\int f(\mathbf{r}, \mathbf{p}) d^{3} p ; \quad \mathbf{j}=\int \mathbf{v} f(\mathbf{r}, \mathbf{p}) d^{3} p,
$$

where the distribution function $f(\mathbf{r}, \mathbf{p})$ is determined by the Vlasov equation

$$
\mathbf{v} \frac{\partial f}{\partial \mathbf{r}}+\frac{\partial}{\partial \mathbf{p}}\left[\left(\mathbf{E}+\mathbf{v} \times \mathbf{B}+\begin{array}{c}
\text { radiation } \\
\text { damping }
\end{array}\right) f\right]=0 .
$$

The velocity $\mathbf{v}$ is given by $\mathbf{v}=\mathbf{p} / \sqrt{\left(\varepsilon^{2}+\mathbf{p}^{2}\right)}$, where the dimensionless parameter $\varepsilon$ is defined by

$$
\varepsilon=\frac{2 m c^{2}}{e B_{0} a^{2} \Omega},
$$

i.e. by the ratio between rest mass energy and homopolar induction energy $\left(B_{0}\right.$ is the polar magnetic field strength, $a$ is the radius and $\Omega$ is the angular velocity of the neutron star). Because typical values for $\varepsilon$ are extremely small $\left(\varepsilon \sim-10^{-12}\right.$ for electrons, $\varepsilon \sim 10^{-9}$ for protons), the particles are expected to become extremely relativistic, since their maximum Lorentz factors should be comparable with $1 /|\varepsilon|$, 
at least if the radiation reaction during phases of acceleration can be neglected. Although this is not the case, as will be discussed in the following, it is nevertheless convenient to use a scaled Lorentz factor $\Gamma=\varepsilon \gamma$ that never exceeds unity.

\subsection{Particle Motion in the Limit of Strong Radiation Damping}

Studying the trajectories of particles in realistic pulsar vacuum fields, one can get an idea of how strongly the radiation reaction does affect the particle motion.

The Lorentz-Dirac equation of motion in the Landau approximation (cf. Landau and Lifshitz 1975) can be written (for $|\varepsilon| \ll 1$ ) as

with

$$
\dot{\mathbf{v}}=\frac{1}{\Gamma} \mathbf{F} ; \quad \dot{\Gamma}=\mathbf{E} \cdot \mathbf{v}-D_{0} \Gamma^{2} \mathbf{F}^{2}
$$

$$
\mathbf{F}=\mathbf{E}+\mathbf{v} \times \mathbf{B}-\mathbf{v}(\mathbf{E} \cdot \mathbf{v}) \text {. }
$$

The dimensionless damping constant $D_{0}$, which is defined by

$$
D_{0}=\frac{e^{2}}{6 \pi \epsilon_{0}} \frac{\Omega}{m c^{3} \varepsilon^{3}},
$$

characterizes the strength of the radiation reaction. For typical pulsar parameters, its value is of the order of $D_{0} \sim-10^{14}$ for electrons, and $D_{0} \sim 10$ for protons. Thus, at least for the electrons, the radiation reaction force dominates the particle motion. Large values of $\left|D_{0}\right|$ imply that the factor of $D_{0}$ in (5a) always remains very small; this leads us to the assumption, which was confirmed by numerical integration of eqs. (5) (cf. Herold et al. 1985, Herold et al. 1986), that during the motion the condition $\mathbf{F} \approx 0$ is fulfilled, which means that the radiation reaction is locally minimized along the trajectory. This is a condition for the velocity and yields, for given $\mathbf{E}$ and $\mathbf{B}$ fields

with

$$
\mathbf{v}=\frac{1}{\mathbf{B}^{2}+P^{2}}\left[\mathbf{E} \times \mathbf{B}+\frac{1}{P}(\mathbf{E} \cdot \mathbf{B}) \mathbf{B}+P \mathbf{E}\right]
$$

$$
P^{2}=\frac{1}{2}\left(\mathrm{E}^{2}-\mathrm{B}^{2}\right)+\frac{1}{2}\left[\left(\mathrm{E}^{2}-\mathrm{B}^{2}\right)^{2}+4(\mathrm{E} \cdot \mathrm{B})^{2}\right]^{\frac{1}{2}} \text {. }
$$

Thereby, we have obtained a local velocity field $\mathbf{v}=\mathbf{v}(\mathbf{E}, \mathbf{B})$ and thus a fluid-like picture for the particle motions in the magnetosphere. The main characteristics of this "damping-free" motion is that the particles try to come to rest in surfaces where $\mathbf{E} \cdot \mathbf{B}=\mathbf{0}$.

\section{NUMERICAL APPROACH AND RESULTS}

Based on these results, the task to determine a self-consistent solution is simpler than before, but still difficult due to the great non-linearity of the problem. Our approach is based on the idea to start from the vacuum solution and to fill up the magnetosphere with the particles that are pulled out from the neutron star 
surface. This is not a real time-dependent calculation, since we assume that the electric field is always described by an electrostatic potential, but for transporting the charge with the velocity (7) we need to solve the time-dependent continuity equation

$$
\dot{\rho}_{e}+\operatorname{div}\left(\rho_{\mathrm{e}} \mathbf{v}\right)=0
$$

and therefore $\dot{E}$ cannot be omitted in Ampere's law. Thus eqs. (1b) have to be replaced by

$$
\left(\Delta-\frac{2}{\rho} \frac{\partial}{\partial \rho}\right)\left(\rho B_{\varphi}\right)=\rho\left(\frac{\partial j_{z}}{\partial \rho}-\frac{\partial j_{\rho}}{\partial z}\right) .
$$

[ Actually, for each particle species the corresponding continuity equation is solved as the velocity (7a) depends on the particle charge: the sign of the quantity $P$ $(P=\mathbf{E} \cdot \mathbf{v})$ from $(7 \mathrm{~b})$ has to be identical to the charge sign. The charge and the current density, which enter Maxwell's equations, are calculated by summation over both particle species. ]

In summary, we solve at each time step the elliptic equations (1a) with Dirichlet boundary conditions for $\Phi$ and $\Psi$ on the star - we assume a homogeneously magnetized neutron star - (the change of $\Phi$ at infinity has to be derived from Ampere's law) and equation (9) with the Neumann boundary condition $\partial\left(\rho B_{\varphi}\right) / \partial r=$ $\rho j_{\vartheta}$ on the star's surface, where the simple emission law $j=\sigma E_{\|}$is assumed. The three elliptic equations are solved by successive over-relaxation (SOR) in a vectorizable checkerboard scheme. For the continuity equations an explicit discretization in time with 2-dimensional Flux Corrected Transport is used in order to preserve steep gradients in the charge density. A more detailed description of our numerical methods is given in Ertl (1988).

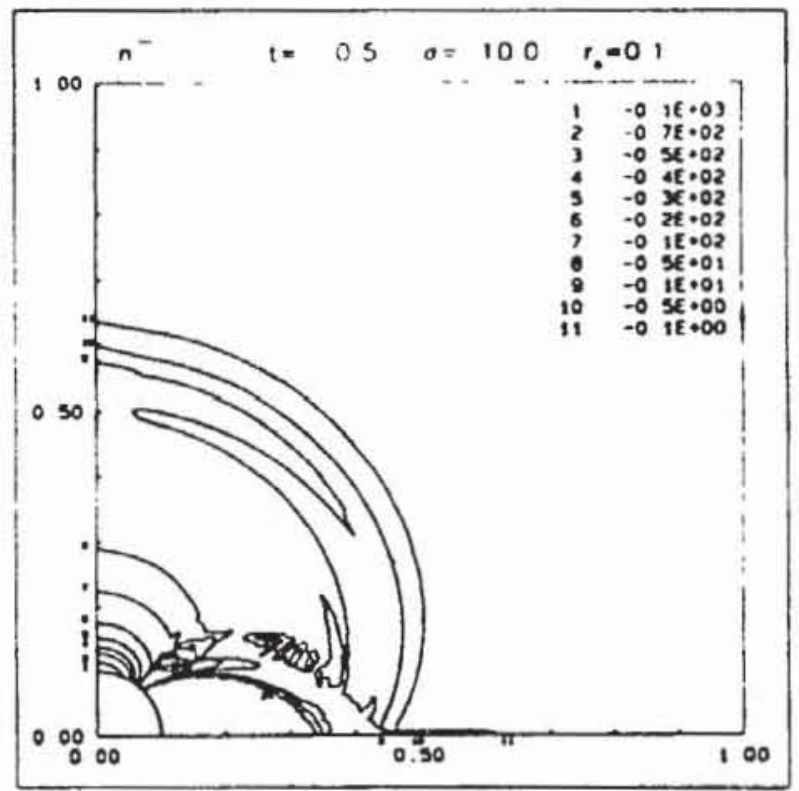

Fig.1: Initial phase: density of the negative particles

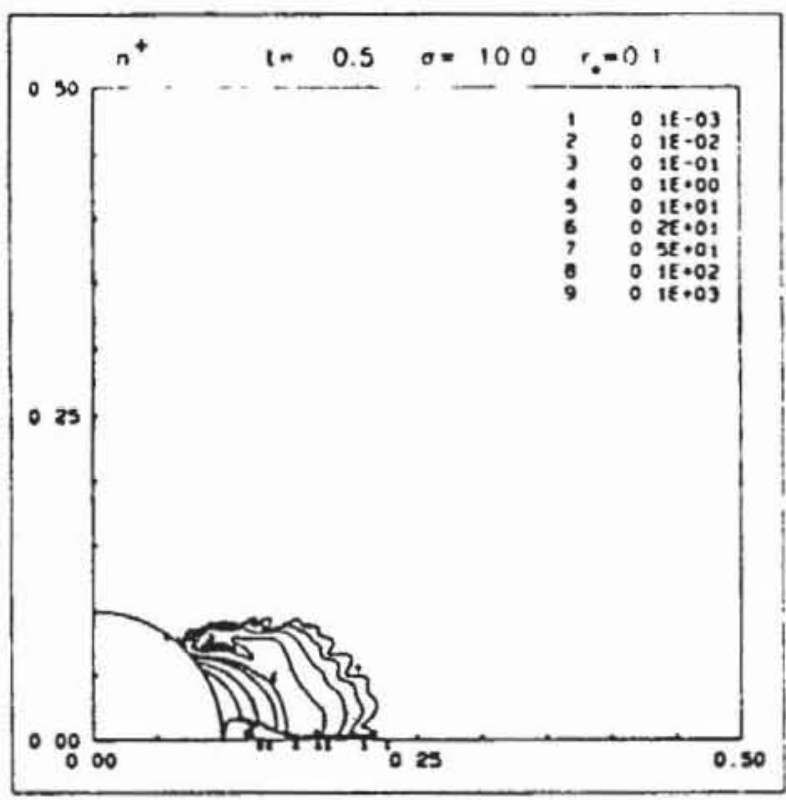

Fig.2: Initial phase: density of the positive particles 
The results described in the following were obtained for a rapid rotator with $r_{0}=(\Omega a) / c=0.1$ as ratio of stellar radius and light cylinder radius (cf. also Ertl et al. 1987). We start with the vacuum fields of an uncharged aligned rotator, i.e. an electric quadrupole field and a magnetic dipole field resulting in $\mathbf{E} \cdot \mathbf{B} \leq 0$ everywhere. Therefore, only negative particles can be emitted and then transported along the magnetic field lines towards the equator where they accumulate. This causes a change in the electric field such that the $\mathbf{E} \cdot \mathbf{B}=0$ surface rises from the equator towards the polar field line forcing the negative particles to follow it. As soon as $\mathbf{E} \cdot \mathbf{B}>0$ at some part of the star's surface positive particles can enter the magnetosphere.

Figs. 1 and 2 show a snapshot of this initial phase at $t=0.5$ (one revolution of the star corresponds to $t=2 \pi$ ). The cloud of positive particles has evolved to a sizable extent, although the negative charge density still dominates. After about one revolution the magnetosphere has developed a structure which already resembles the final situation. This quasi-stationary end phase is depicted (after 35 revolutions) in Figs. $3-6$, where the charge densities and the contour lines of the electrostatic potential and of $\mathbf{E} \cdot \mathbf{B}$ are shown. One recognizes two regions of charged particles (the negative ones in a cone around the polar field line, the positive ones around the equatorial plane) separated by a vacuum gap in between. (Vacuum gaps in non-selfconsistent models are not new; for a more recent model with such a feature, cf. Mestel et al. 1985.) In this vacuum gap the parallel electric field $E_{\|}=(\mathrm{E} \cdot \mathrm{B}) /|\mathrm{B}|$ is greatly different from zero (s. Fig. 6), whereas in the regions populated with plasma the electric field, which is now totally unsimilar to the initial quadrupole (s. Fig. 5), has evolved as to achieve $\mathbf{E} \cdot \mathbf{B} \sim 0$ there. The poloidal magnetic field does not change very much, it remains essentially dipolar. The neutron star is now positively charged, and the positive and the negative currents out of the star exactly match each other.

From the velocity fields - which are not shown here - one can conclude that the positive particles corotate with the star (to a very good approximation) as long as they are inside the light cylinder, but close to the light cylinder they lose corotation and stream through a sort of nozzle into the outer parts of the magnetosphere forming a radial wind. The velocity of the negative particles is dominantly poloidal and, thus, also a negatively charged wind builds up.

There are still some wave-like fluctuations, especially inside the negative zone, which seem to originate from the tendency of the system to make $\mathbf{E} \cdot \mathbf{B}$ small (or even zero) in the plasma regions. This also explains the strange folded structure of the $\mathbf{E} \cdot \mathbf{B}=0$ surface and might be an indication that the magnetosphere wants to form an extended region with $\mathbf{E} \cdot \mathbf{B}=0$; however, this is difficult to achieve with a relativistic particle population. Whether for this end a cool non-relativistic plasma population is necessary or whether such fluctuations are a physically real part of a quasi-stationary configuration is an open question. Globally, our solution represents a stationary structure; for instance, the charges of the star and of the magnetosphere remain, in the end phase, constant with great accuracy.

For the first time in pulsar magnetospheric theory it seems that a stationary self-consistent solution for the aligned rotator has been found by a quasi-timedependent simulation. Detailed diagnostic investigations of this solution, such as the calculation of the radiation of the accelerated particles, as well as studies of 


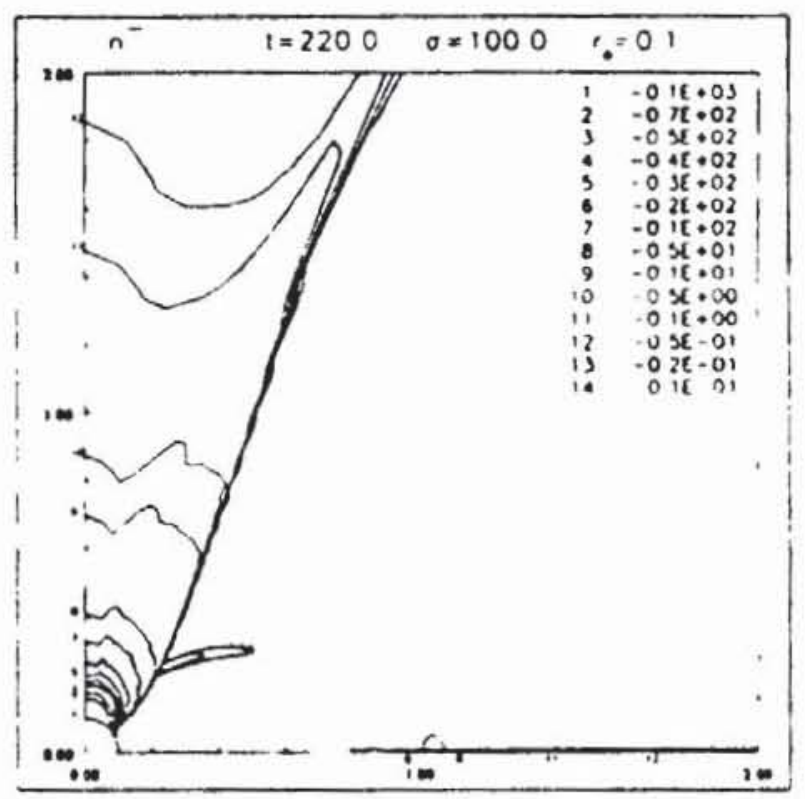

Fig.3: Quasi-stationary end phase: density of the negative particles

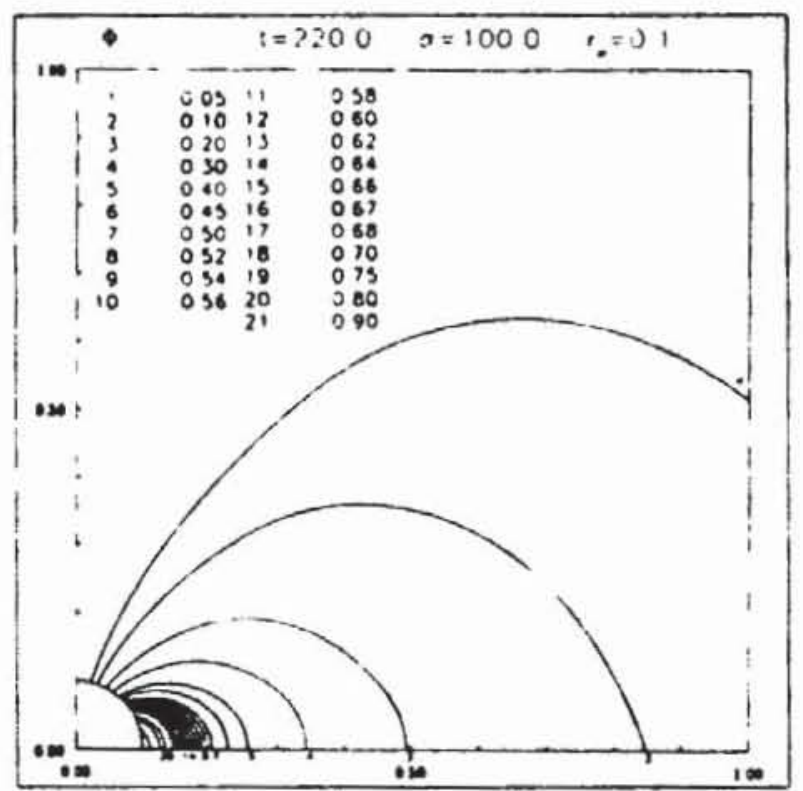

Fig.5: Quasi-stationary end phase: contour lines of the electrostatic potential $\Phi$

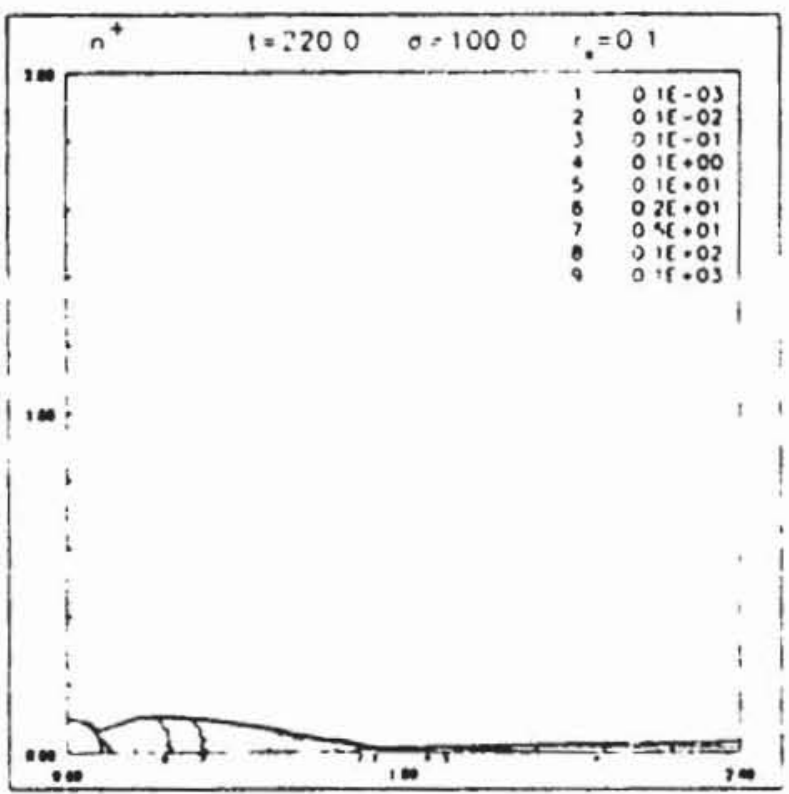

Fig.4: Quasi-stationary end phase: density of the positive particles

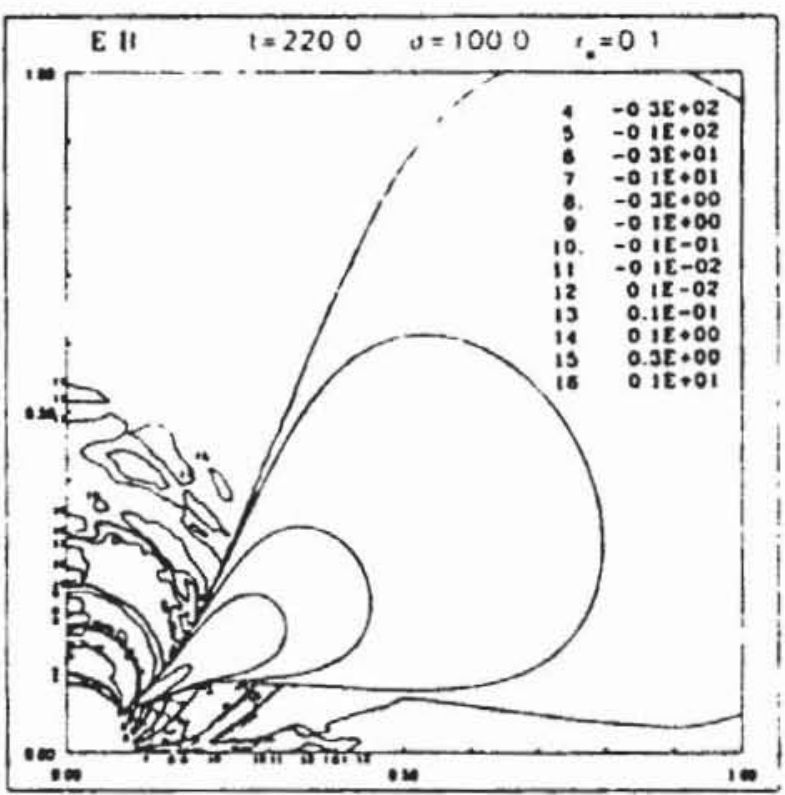

Fig.6: Quasi-stationary end phase: contour lines of $\mathbf{E} \cdot \mathbf{B}$

the parameter dependence (rotation frequeny $\Omega$, emissivity $\sigma$ of the neutron star) are tasks for the future.

\section{REFERENCES}

Backer, D.: 1989 , in these proceedings

Ertl, T.: 1988, Thesis, Universität Tübingen 
Ertl, T., Herold, H., Finkbeiner, B., Ruder, H.: 1987, Mitt. Astron. Ges. 70, 372

Goldreich, P., Julian, W.H.: 1969, Astrophys. J. 157, 869

Herold, H., Ertl, T., Ruder, H.: 1985, Mitt. Astron. Ges. 63, 174

Herold, H., Ertl, T., Ruder, H.: 1986, Proceedings of the 8th EPS Conference on Computational Physics - Computing in Plasma Physics, Eibsee, Europhysics Conference Abstracts 10D, 3

Landau, L.D., Lifshitz, E.M.: 1975, The Classical Theory of Fields, Pergamon Press, Oxford

Mestel, L.: 1981, in "Pulsars", IAU Symp. 95, Reidel, Dordrecht, p. 37

Mestel, L., Robertson, J.A., Wang. Y.-M., Westfold, K.C.: 1985, Mon. Not. R. Astr. Soc. 217, 443

Michel, F.C.: 1982, Rev. Mod. Phys. 54, 1 